\title{
Estimating heritability in a threshold trait: heat-shock tolerance in Drosophila buzzatii
}

\author{
ROBERT A. KREBS* \& VOLKER LOESCHCKE \\ Department of Ecology and Genetics, University of Aarhus, Ny Munkegade, 8000 Aarhus C, Denmark
}

\begin{abstract}
Stress tolerance is often measured as a threshold trait, the proportion of a group that survives a defined stress regime. Requirements of large offspring numbers coupled with fitness variation in the surviving cohort limit the use of some standard genetic analyses for estimating heritability. Therefore, we present an isofemale line analysis, which is a modified full-sib design, to estimate heritability of tolerance to heat shock in pretreated Drosophila buzzatii adults. Highly significant levels of genetic variation were found in males and females at the third generation of laboratory rearing, and the intraclass correlations were estimated to be about 0.2 for four independent sets of 25 isofemale lines. The proportion of the variance explained within lines among same-sex replicates, however, was larger than that between replicates of males and females. Because genetic variation was estimated from groups, the error variation required factoring by the group size to estimate heritability, which averaged 0.03 . The four most tolerant, four least tolerant and four lines of average tolerance to heat stress in each set were reanalysed after $10-11$ generations of rearing at $25^{\circ} \mathrm{C}$. Survival in the 13 th-14th generations was positively and significantly associated with survival at generation 3 . These comparisons indicate the high repeatability of measurements of heat-shock tolerance.
\end{abstract}

Keywords: heritability, isofemale lines, quantitative genetics, repeatability, thermotolerance.

\section{Introduction}

Tolerance to environmental extremes may affect species distribution and abundances. For sexually reproducing species, high levels of genetic variation for environmental tolerance may be associated with colonizing ability (Parsons, 1983) or the ability of a population to adapt to changing conditions (Parsons, 1973; Huey \& Bennett, 1990; Hoffmann \& Parsons, 1991, pp. 58-75). Similarly, for parthenogenetic clones, genetic differences may influence which individuals survive to colonize novel habitats (Weider, 1993; Niklasson \& Parker, 1994). The genetics of high temperature (or heat shock) tolerance is not easily studied, because survival following a stress is a threshold trait with an underlying variable that is usually assumed to be normally distributed (Falconer, 1981). Furthermore, even within populations of one species, Drosophila melanogaster, the amount of genetic variation reported (Parsons, 1989;

*Correspondence: Department of Organismal Biology and Anatomy, The University of Chicago, 1027 East 57th Street, Chicago, Illinois 60637, U.S.A. E-mail: r-krebs@uchicago.edu
Huey et al., 1992; Jenkins \& Hoffmann, 1994; Krebs \& Loeschcke, 1994a) may vary considerably.

Knowledge of the genetic basis of threshold traits lags behind that for meristic traits. The threshold trait, survival after heat stress, is estimated on the basis of group data, and is sensitive to small differences in temperature (Krebs \& Loeschcke, 1994b). Selection on survival can produce divergent lines in stress tolerance (e.g. Morrison \& Milkman 1978; Kilias \& Alahiotis 1985; Quintana \& Prevosti 1990), but technical problems have often obscured the estimation of heritability (Krebs \& Loeschcke, 1996).

We examined tolerance to heat shock (as the proportion surviving) among defined sets of isofemale lines. These are lines derived from singly inseminated females, and they provide useful information about variance components of quantitative traits (Parsons, 1980; Hoffmann \& Parsons, 1988; David et al., 1994). Genetic variation within isofemale lines is $3 / 4$ the additive genetic variance $\left(V_{\mathrm{A}}\right)$, or that for full-sibs after one generation of brothersister mating, provided that lines are reared in large numbers and that lines are analysed within a few 
generations in the laboratory (Hoffmann \& Parsons, 1988). Genetic variation in stress tolerance within populations is predominantly additive (Hoffmann \& Parsons, 1991; McColl et al., 1996), although dominance or epistatic effects may explain some differences in stress tolerance between populations of both $D$. melanogaster and D. buzzatii (Krebs et al., 1996). If present in an isofemale line analysis, these effects will cause an underestimation of heritability in the broad sense and an overestimation in the narrow sense (Hoffmann \& Parsons, 1988). Therefore, although isofemale line techniques make tractable the analysis of some traits that are otherwise difficult to measure, a loss of precision may result.

Here, we ask, for heat-shock tolerance, how large is the genetic component of variation among isofemale lines, and are differences consistent after a further $10-11$ generations of rearing at $25^{\circ} \mathrm{C}$ ? To strengthen the estimates of genetic variation, analyses were derived from four independent sets of lines drawn from one base population. Technical difficulties in replicating stress levels were compensated statistically by measuring thermotolerance as the residual survival after analysis of day-to-day variation in the stress treatment. Results compared repeatability of thermotolerance among replicates of the same line within a single generation, and the mean thermotolerance of lines separated by 10-11 generations' rearing at $25^{\circ} \mathrm{C}$. Individual heritability was estimated following Hoffmann \& Parsons (1988), but included a slight modification of the method to estimate density dependence in the group error term more precisely.

\section{Materials and methods}

Drosophila buzzatii were collected from southwestern Tenerife, Canary Islands (Lat. $28^{\circ} 10^{\prime} \mathrm{N}$ ), in December 1993. More than 80 wild-caught $D$. buzzatii were collected and pooled in one chamber for 5 days to permit intermating, and were afterwards distributed among four $200-\mathrm{mL}$ flasks containing instant Drosophila medium (Carolina Biological Supply). Virgin males and females were collected from these flasks under $\mathrm{CO}_{2}$ anaesthesia. One male and one female were placed in each of 110 vials (also on instant medium) to establish isofemale lines. Those lines were maintained where more than 40 offspring emerged, a condition satisfied by more than the 100 lines needed for the experiments. Therefore, selection on fitness in the laboratory environment, which would have affected betweenline variance, was minimized. The offspring from these 100 lines were transferred to four vials per line, with a minimum of five males and five females per vial as a procedure for maintenance. Lines were assigned to four independent sets of 25 lines each, which were separated in development by $3-5$ days to facilitate maintenance and experimental handling. Second-generation adults were collected similarly, distributed among four vials and transferred to new vials every 2 days over a 6-day period. Thereby, 12 vials per line were available for collecting experimental flies for heat shock, and the last transfer was used to continue lines. All maintenance and growth occurred in a $25^{\circ} \mathrm{C}, 70$ per cent relative humidity environmental room.

To analyse heat-shock tolerance of adult $D$. buzzatii, virgin males and females were pretreated to condition individuals to heat by exposure to $38^{\circ} \mathrm{C}$ (incubator temperature) for $75 \mathrm{~min}$. After $24 \mathrm{~h}$, these flies were exposed to a severe temperature stress of $41.5^{\circ} \mathrm{C}$ (incubator temperature) for $100 \mathrm{~min}$. Actual temperatures for pretreatment and heat shock were a little lower than incubator temperatures and were described in more detail previously, along with the detailed description of the methods for inducing heat stress (Krebs \& Loeschcke, 1994a,b). To perform these stress experiments, flies of each line were separated by sex and placed in groups of 20 per vial, which contained a sucrose-agar-yeast medium with live yeast added to the surface. Fresh vials were provided after 3 days, which was immediately before the pretreatment. After both treatments, all flies recovered in a $25^{\circ} \mathrm{C}$ environmental room.

Heat-shock tolerance in the four sets of 25 lines was analysed separately. For each set, flies were collected and treated in six blocks of replicates, with each block containing one vial of males and one vial of females per line, with all flies of the same age. These 50 vials were randomized within a large rack during pretreatment and heat shock. Data were the proportion of individuals able to walk $24 \mathrm{~h}$ after exposure to stress, which initially immobilizes adults.

Heat-shock tolerance was retested after 6 months' rearing at $25^{\circ} \mathrm{C}$. This time period was 10 generations for two sets of lines and 11 generations for the other two sets. Half the 12 lines from each set were retested, and these were chosen based on the tolerance to heat stress in the first experiment. Retested were the four most tolerant, the four least tolerant and four that were of average tolerance. Equal weight was given to tolerance of males and females when choosing lines. Heat-shock tolerance was measured identically to that in the first analyses, and each set of lines was again analysed separately from all the other sets. With the reduced number of lines, two 
vials of males and two vials of females were used in each of four replicate blocks of experiments.

\section{Genetic analysis and statistics}

Thermotolerance was scored as the proportion of surviving adults in each vial, and this value was arcsine-square root transformed. Using these transformed survival values, we analysed additive genetic variance components $\left(S_{\mathrm{A}}^{2}\right)$ for each set of lines, and for males and females separately. Thermotolerance of males typically exceeds that of females in $D$. buzzatii (Loeschcke et al., 1994). $S_{\mathrm{A}}^{2}$ is obtained from a two-way ANOVA of the block and line effects without interaction (as this interaction is the error component) for each set of lines. Starting with the mean square of the line effect, the MSE is subtracted, and this difference is divided by the number of replicates (six vials per line at generation 3 and eight vials in the retest of lines). The intraclass correlation $(t)$ is subsequently estimated by $S_{\AA}^{2} /\left(S_{\AA}^{2}+\mathrm{MSE}\right)$.

As $t$ was estimated from groups of 20 individuals per vial, estimates of the isofemale line heritability $(H)$, which is the same as the intraciass correlation, $t$, when each individual is measured, required correction for group size $(x)$. Hoffmann \& Parsons $\left(1988\right.$, p. 93) proposed $S_{\mathrm{A}}^{2} /\left(S_{\mathrm{A}}^{2}+x s^{2}\right)$ for this correction, where $s^{2}$, the variance component within lines, is MSE in the present analyses. This correction is sufficient if there is no nongenetic correlation among the different individuals within groups, of which common environment effects is one possibility (discussed in Mather \& Jinks, 1977, p. 53). If a correlation is present but not detected, within-line variance and the intraclass correlation coefficient will be overestimated. However, our estimate of heritability may be either overestimated because of an inflated $S_{\mathrm{A}}^{2}$, or underestimated because too large a fraction of the MSE will be multiplied by the group correction factor. Underestimation of heritability is more likely where common environment effects are small.

Therefore, we estimate heritability from group means after replacing the between-line and withinline variances, $E_{\mathrm{b}}$ and $E_{\mathrm{w}}$, for total variance, using terminology from Mather \& Jinks (1977). Only within-line variance, which is equivalent to MSE $-E_{\mathrm{b}}$, is subject to group size effects. Substituting $E_{\mathrm{b}}+x E_{\mathrm{w}}$ in the equation estimating isofemale heritability, $H=S_{\mathrm{A}}^{2} /\left(S_{\mathrm{A}}^{2}+x \mathrm{MSE}\right)$ of Hoffmann \& Parsons (1988, p. 93), for their $x$ MSE gives, with rearrangement, $H=S_{\mathrm{A}}^{2} /\left[S_{\mathrm{A}}^{2}+E_{\mathrm{b}}+x\left(\mathrm{MSE}-E_{\mathrm{b}}\right)\right]$. The two components of error can easily be estimated by plotting the mean square error from independent analyses of variance against the inverse of group size. A simple linear equation $[y=a+b(1 / x)]$ is obtained, where the intercept estimates $E_{\mathrm{b}}$, the slope estimates $E_{\mathrm{w}}$, and the intragroup correlation, $r=a /(a+b)$. To estimate $r$, we took data (from Malmberg, 1994) on four group sizes for each sex: 5 , 10,15 or 20 males or females to compare the contributions of $E_{\mathrm{b}}$ and $E_{\mathrm{w}}$ to the error variance.

As a second test of genetic differences within and among lines, we compared genetic differences in variation in thermotolerance of males and females within the same generation and across 6 months of rearing lines at $25^{\circ} \mathrm{C}$. Block variation is inherent in the heat-shock methodology owing to day-to-day variation in either the stress temperature or the rate of temperature increase within the incubator, and this variation can obscure genetic differences among lines. To homogenize means across sets of lines and blocks of replicates, all data were used in an analysis of variance, whereby set effects and block-nested within-set effects were defined, with separate analyses for males and females. The magnitude of residuals from this initial analysis provides a thermotolerance score for each line, which is easily interpreted as positive scores indicating higher survival than the population mean, and negative scores indicating lower survival. The proportion of variance explained by line effects across generations and by similarities between different-sex offspring was estimated by correlation analysis of these mean line scores. Thereby, each set of lines contributes equal weight when examining genetic relationships among males and females for thermotolerance and for comparisons of thermotolerance after 10-11 generations of laboratory rearing.

\section{Results}

\section{Genetic variation}

The significance of the line effect in seven of eight analyses of line variation, which apply to the variance component between strains, $S_{\mathrm{A}}^{2}$, indicated that heat-shock tolerance is genetically variable in this $D$. buzzatii population (Table 1). The intraclass correlations were estimated for each sex and set using the mean square errors indicated. Because the four sets of lines were drawn as random samples from the same mass population, means for the population, and standard errors of these means, were computed from each individual estimate. The line component of genetic variance, which is the intraclass correlation, $t$, gave a mean for $t$ of 0.22 among brothers and 
Table 1 The proportion of additive genetic variation, $S_{A}^{2}$, for heat-stress tolerance of Drosophila buzzatii males and females, the mean square error (MSE) of the analyses of variance from which genetic variation was estimated and the intraclass correlation, $t$

\begin{tabular}{cllllll}
\hline Sets & & Males & Females & Sets & Males & Females \\
\hline 1 & $S_{\mathrm{A}}^{2}$ & 0.0008 & $0.0050^{*}$ & 3 & $0.0219^{* * *}$ & $0.0043^{*}$ \\
& MSE & 0.0656 & 0.0496 & & 0.0431 & 0.0378 \\
& $t$ & 0.012 & 0.092 & & 0.337 & 0.102 \\
& $H$ & 0.0006 & 0.0051 & & 0.0248 & 0.0056 \\
& $h^{2}$ & 0.0012 & 0.0101 & & 0.0502 & 0.0113 \\
2 & $S_{\mathrm{A}}^{2}$ & $0.0158^{* * *}$ & $0.01275^{* * *}$ & 4 & $0.0211^{* * *}$ & $0.0187^{* * *}$ \\
& MSE & 0.0416 & 0.0447 & & 0.0596 & 0.0384 \\
& $t$ & 0.275 & 0.222 & & 0.262 & 0.328 \\
& $H$ & 0.0186 & 0.0141 & & 0.0174 & 0.0238 \\
& $h^{2}$ & 0.0376 & 0.0283 & & 0.0351 & 0.0482 \\
Mean & & & & & & \\
& $t$ & & & & $0.222 \pm 0.072$ & $0.186 \pm 0.028$ \\
& $H$ & & & & $0.015 \pm 0.005$ & $0.012 \pm 0.004$ \\
& $h^{2}$ & & & $0.031 \pm 0.010$ & $0.024 \pm 0.009$ \\
\hline
\end{tabular}

Significance was tested by the line component from analyses of variance. The isofemale heritability $(H)$ and narrow-sense heritability $\left(h^{2}\right)$ were estimated from the intraclass correlation.

Significance from zero: ${ }^{*} P<0.05 ;{ }^{* *} P<0.001$.

of 0.19 among sisters (Table 1), meaning that genetic differences among lines account for about 20 per cent of the variation in survival after removing block effects. This difference in $t$ between males and females was not significant. However, the correlation between the survival of males and females was only 0.34 (Fig. 1). Although this correlation was significantly different from zero $(P<0.001), r^{2}$ for this estimate of between-sex variation caused by genetic differences among lines was only 0.11 , which was half that for within-sex variation.

\section{Estimating heritability from groups}

The first step in estimating an individual's contribution to isofemale heritability was to determine the influence of group size $(x)$ on the error variance in ANOVAs. Neither slopes nor intercepts differed for males and females, and the regression line of $1 / x$ against MSE for pooled data provided estimates of the intercept, $E_{\mathrm{b}}$, of $0.008 \pm 0.010$ and of the slope, $E_{\mathrm{w}}$, of $0.61 \pm 0.08$. Because only the effects on $E_{\mathrm{w}}$, the within-line variation, were significantly different from zero, and not those on $E_{\mathrm{b}}$, or that between lines, the intragroup correlation was also not significantly different from zero. The assumption of no nongenetic effects on between-line variance was therefore satisfied, which enables use of the simple correction factor of Hoffmann \& Parsons (1988) of 20 for all group means (Table 1). However, we note that, as the proportion of the variation resulting from $E_{\mathrm{b}}$ is much smaller than that resulting from $E_{\mathrm{w}}$ (to which each individual contributes), misapplying variance to or from $E_{\mathrm{b}}$ can have larger effects on isofemale heritability than will error in $E_{\mathrm{w}}$, especially with larger group sizes.

Estimates of isofemale heritability and the narrow-sense heritability (assuming no dominance effects) from the intraclass correlations were small (Table 1), despite being measured from significant components of variance (except set 1 males, in which variation was not significant). Males and females were not significantly different in heritability of heat-shock tolerance (Table 1).

\section{Cross-generation correlations}

Differences among lines for survival after heat stress of males and females persisted through a retest of a proportion of the original lines from each set, the four most resistant, the four least resistant and the four of average tolerance, after maintenance at $25^{\circ} \mathrm{C}$ for 10-11 additional generations (Table 2). Intraclass correlations were higher than those presented in Table 1, and results fluctuated within sets, increasing or decreasing between analyses, but no 
significant differences occurred for the means of intraclass correlations either of males or of females measured 10-11 generations apart (Table 2). The higher correlations were expected, as many intermediate lines were omitted from the reanalysis.

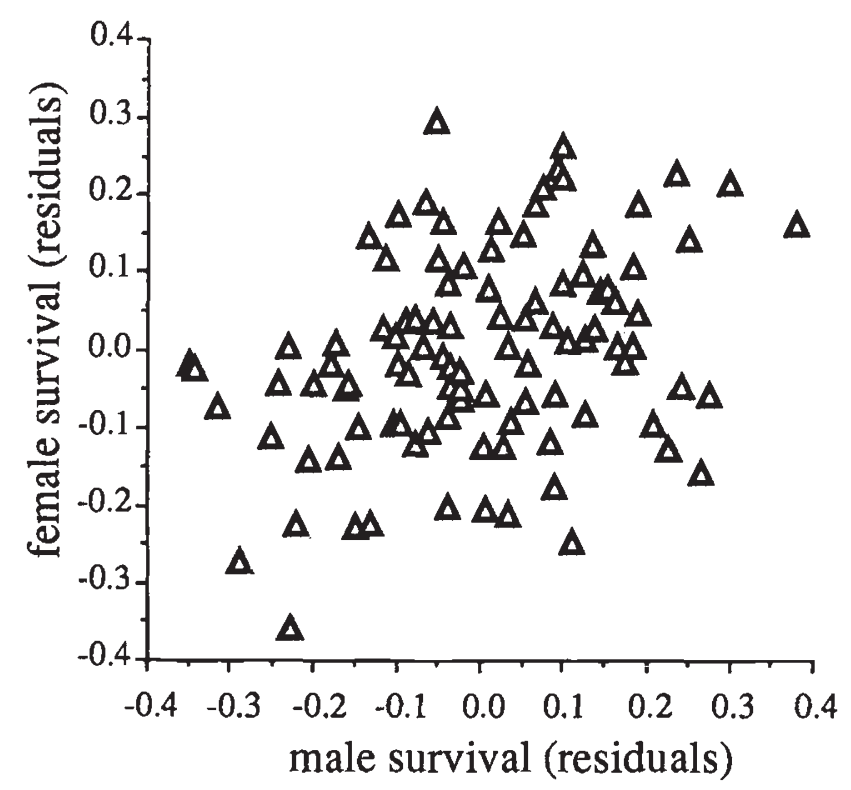

Fig. 1 The relationship between Drosophila buzzatii males and females for survival after heat shock, shown as a plot of mean female tolerance of each line against mean male tolerance of each line. Because 100 isofemale lines were analysed in four sets of 25 lines each, with six replicate blocks per set, general linear models were used first to partition set and block-within-set effects. The mean residuals for each line from these separate ANOVAs on male and female survival are plotted $(r=0.34, P<0.001)$.
Higher survival in generation 3 was associated with higher survival in generations 13 and 14 (Fig. 2). This association was greater in females (explaining 41.6 per cent of the variance between experiments) than in males (18.0 per cent of the variance explained). In contrast to results within a generation, these correlations between generations are far more likely to be reduced than increased by non-genetic effects that may have affected betweenline variation. Actual survival rates to the stress treatment differed between the original analysis and the retest. For only those lines that were retested, mean survival to stress was similar between the original analysis $( \pm 1 \mathrm{SE}$, with standard errors estimated from block means), in which $36 \pm 3$ per cent survived, and the retest, in which $43 \pm 3$ per cent. However, male and female differences for survival after stress were greater in the retest, with that of males rising from 42 per cent to 60 per cent with maintenance in the constant environment, and tolerance of females decreasing from 30 per cent to 26 per cent under these treatment conditions.

\section{Discussion}

Genetic variation among isofemale lines of D. buzzatii explains a large proportion of the variance in mean heat-shock tolerance of pretreated adults. Furthermore, same-sex siblings may be genetically more similar than opposite-sex siblings for heatshock tolerance, suggesting that male and female Drosophila differ in the mechanistic basis for thermotolerance. Some loci that contribute to genetic variation in heat-shock tolerance may be either-sex specific or their effects may predominate in one sex or the other (Krebs et al., 1996). This possibility is

Table 2 Estimates of the intraclass correlation, $t$, from a retest of male and female tolerance to heat shock in 48 Drosophila buzzatii lines, 12 from each of four sets

\begin{tabular}{lcccccc}
\hline Experiment & Sets & Males & Females & Sets & Males & Females \\
\hline Retest & 1 & $0.492^{* * *}$ & $0.393^{* * *}$ & \multirow{2}{*}{3} & $0.313^{* * *}$ & $0.329^{* * *}$ \\
Original & & 0.127 & $0.317^{* *}$ & & $0.540^{* * *}$ & $0.214^{*}$ \\
Retest & 2 & $0.136^{*}$ & $0.294^{* * *}$ & 4 & $0.386^{* * *}$ & $0.408^{* * *}$ \\
Original & & $0.333^{* *}$ & $0.489^{* * *}$ & & $0.316^{* *}$ & $0.510^{* * *}$ \\
Retest means & & & & & $0.332 \pm 0.075$ & $0.356 \pm 0.027$ \\
Original means & & & & & $0.329 \pm 0.084$ & $0.382 \pm 0.071$ \\
\hline
\end{tabular}

Intraclass correlations for the original results on the 12 lines used in the retest are also presented. Significance was tested by the line component from analyses of variance.

Significance from zero: ${ }^{*} P<0.05 ;{ }^{*} P<0.01 ;{ }^{* * *} P<0.001$. 
supported by differences in tolerance between male and female Drosophila in many species (Maynard Smith, 1957; Milkman, 1963; Jenkins \& Hoffmann, 1994; Loeschcke et al., 1994), by sex differences in cross-environment interactions with sex (Dahlgaard
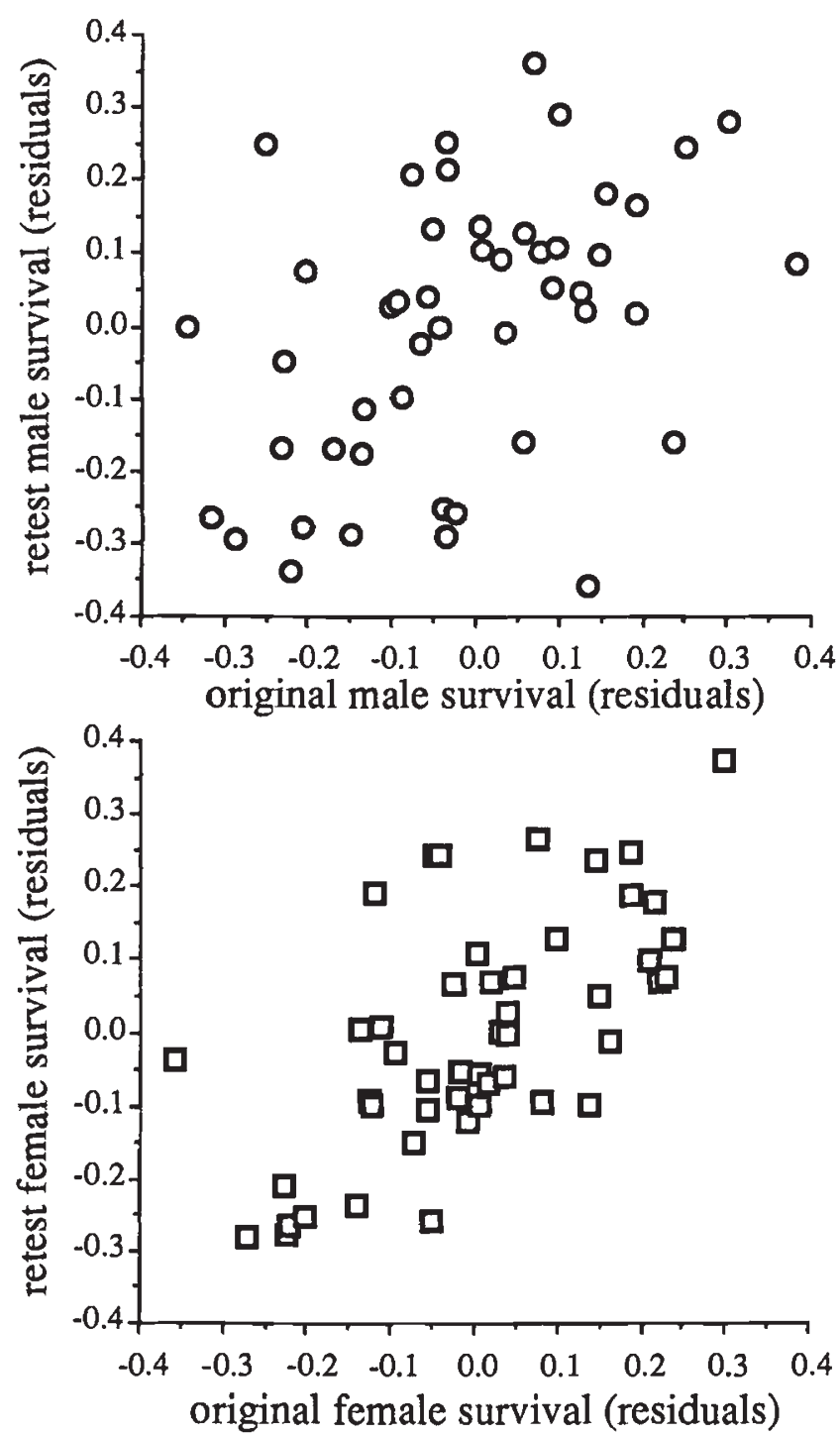

Fig. 2 The relationship for Drosophila buzzatii between male and female tolerance of heat shock at generation 3 and, following rearing at constant $25^{\circ} \mathrm{C}$, thermotolerance at generations 13-14. Because 100 isofemale lines were originally analysed in four sets of 25 lines each, and 12 lines from each set were retested, general linear models were used to partition set and block-within-set effects. The mean residuals for each line that were generated from these separate ANOvas on male and female survival at each time point are plotted. The original results explained 18.0 per cent of the variance in survival of males and 41.6 per cent of the variance in survival of females between analyses. et al., 1995) and by differential response to selection for increased tolerance between males and females (Krebs \& Loeschcke, 1996).

Despite a high proportion of the variance that is explained by the line effect and a corresponding high group heritability, as defined by the intraclass correlation, each individual contributes little to the probability of its offspring surviving thermal stress. Similarly, low heritability was suggested from slow selection responses for increased heat-shock tolerance in D. buzzatii (Krebs \& Loeschcke, 1996) and in D. melanogaster (McColl et al., 1996), under conditions where all adults are pretreated before selection. Heritability estimates where adult Drosophila are not pretreated to induce high thermotolerance provide a mixture of results, some of low estimates (Morrison \& Milkman, 1978; Quintana \& Prevosti, 1990) and others high (Huey et al., 1992; Jenkins \& Hoffmann, 1994).

Low estimates of heritability for a trait with high group repeatability over time result from the greater precision in estimating group means than is possible for any particular measurement on an individual. Hence, the probability of surviving heat shock may be accurately estimated for lines of Drosophila even though an individual's phenotype is alive or dead. Chance plays a larger role in predictions of an individual's survival than the mean of a group. However, an individual's contribution to the error variance can be factored back into results for group heritabilities (Hoffmann \& Parsons, 1988), provided that the relative contribution of group size-dependent and -independent effects must first be estimated by determining the intragroup correlation (as described here in Methods). The requirement of first estimating group size effects, or particularly the density-independent variation within groups, is only a minor addition to the isofemale line technique and is likewise necessary for conventional full-sib or half-sib designs (Mather \& Jinks, 1977). The experimental factors most likely to contribute to the intragroup correlation, for example common rearing environment or spatial variation in the stress chamber, can be estimated in a preliminary experiment, as carried out here.

The isofemale line design provides the unique benefit that, after estimating the heritability of heatshock tolerance, lines of known tolerance levels remain available for subsequent analyses of correlated traits. To compare relationships among experiments in different generations, however, variation must be repeatable. Here, we found that, even for freshly collected lines, relatively high or low tolerance to heat stress persists over many generations. 
Repeatability of heat-shock tolerance will be affected by drift and inbreeding (Maynard Smith, 1956; Dahlgaard et al., 1995), but within each line only alleles at polymorphic loci change frequency. Specific traits may change little in a large proportion of lines, because homozygous loci that either positively or negatively influence heat-shock tolerance maintain this influence over many generations, and may dominate changes at variable loci. Empirical evidence for repeatability is limited, however, with Da Lage et al. (1990) finding repeatability to be low for desiccation tolerance, Hosgood \& Parsons (1968) observing significant changes in tolerance to heat in a small number of lines, whereas Barker (1992) observed high repeatability for oviposition preference. Here, we found that, even for freshly collected lines, relatively high or low tolerance to heat stress persists over many generations.

\section{Acknowledgements}

We thank Camilla Håkansson and Doth Andersen for their valuable assistance with data collection, Ary Hoffmann for discussion of the group measurement problem and Kuke Bijlsma, Jesper Dahlgaard and Jürgen Tomiuk for comments on the manuscript. This research was made possible by grants from the Carlsberg Foundation (No. 93-0280-30) and the Danish Natural Science Research Council (Nos 94-0163-1 and 11-0533-01).

\section{References}

BARKER, I. S. F. 1992. Genetic variation in cactophilic Drosophila for oviposition on natural yeast substrates. Evolution, 46, 1070-1083.

DAHLGAARD, J., KREBS, R. A. AND LOESCHCKE, V. 1995. Heat-shock tolerance and inbreeding in Drosophila buzzatii. Heredity, 74, 157-163.

DA LAGE, J. L., CAPY, P. AND DAVID, J. R. 1990. Starvation and desiccation tolerance in Drosophila melanogaster: differences between European, North African and Afrotropical populations. Génét. Sél. Évol., 22, 381-391.

DAViD, J. R., MORETEAU, B., GAUThIER, J. P., PETAVy, G., STOCKEL, A. AND IMASHEVA, A. G. 1994. Reaction norms of size and shape characters in relation to growth temperature in Drosophila melanogaster: an isofemale line analysis. Génét. Sél. Évol., 26, 229-251.

FALCONER, D. S. 1981. Introduction to Quantitative Genetics, 2nd edn. Longman, London.

HOFFMANN, A. A. AND PARSONS, P. A. 1988. The analysis of quantitative variation in natural populations with isofemale strains. Génét. Sél. Évol., 20, 87-98.
HOFFMANN, A. A. AND PARSONS, P. A. 1991. Evolutionary Genetics and Environmental Stress. Oxford Scientific, Oxford.

HOSGOOD, s. M. W. AND PARSONS, P. A. 1968. Polymorphism in natural populations of Drosophila for the ability to withstand temperature shocks. Experientia, 24, 727-728.

HUEY, R. B. AND BENNETT, A. F. 1990. Physiological adjustments to fluctuating thermal environments: an ecological and evolutionary perspective. In: Morimoto, R. I., Tissiéres, A. and Georgopoulos, C. (eds) Stress Proteins in Biology and Medicine, pp. 37-59. Cold Spring Harbor Press, Cold Spring Harbor, NY.

HUEY, R. B., CRILl, W. D., KINGSOlVER, J. G. AND WEBER, K. E. 1992. A method for rapid measurement of heat or cold resistance of small insects. Funct. Ecol., 6, 489-494.

Jenkins, N. L. AND hofFmann, A. A. 1994. Genetic and maternal variation for heat resistance in Drosophila from the field. Genetics, 137, 783-789.

KILIAS, G. AND ALAHIOTIS, S. N. 1985. Indirect thermal selection in Drosophila melanogaster and adaptive consequences. Theor. Appl. Genet., 69, 645-650.

KREBS, R. A. AND LOESCHCKE, V. 1994a. Response to environmental change: genetic variation and fitness in Drosophila buzzatii following temperature stress. In: Loeschcke, V., Tomiuk, J. and Jain, S. K. (eds) Conservation Genetics, pp. 309-321. Birkhäuser, Basle.

KREBS, R. A. AND LOESCHCKE, V. 1994b. Effects of exposure to short-term thermal extremes on fitness components in Drosophila melanogaster. J. Evol. Biol., 7, 39-49.

KREBS, R. A. AND LOESCHCKE, V. 1996. Acclimation and selection for increased resistance to thermal stress in Drosophila buzzatii. Genetics, 142, 471-479.

KREBS, R. A., LA TORRE, V., LOESCHCKE, V. AND CAVICCHI, s. 1996. Heat-shock resistance in Drosophila populations: analysis of variation in reciprocal cross progeny. Hereditas, 124, 47-55.

LOESCHCKE, V., KREBS, R. A. AND BARKER, J. S. F. 1994. Genetic variation for resistance and acclimation to high temperature stress in Drosophila buzzatii. Biol. J. Linn. Soc., 52, 83-92.

McCOlL, G., HOFFmANN, A. A. AND McKeChNIE, s. W. 1996. Response of two heat shock genes to selection for knockdown heat resistance in Drosophila melanogaster. Genetics, 143, 1615-1627.

malmberg, L. 1994. The Effects of Life History on High Temperature Tolerance in Drosophila buzzatii. M.Sc. Thesis, Lund University, Sweden.

MATHER, K. AND JINKS, J. L. 1977. Introduction to Biometrical Genetics, 3rd edn. Chapman \& Hall, London.

MAYNARD SMITH, J. 1956. Acclimatization to high temperatures in inbred and outbred Drosophila subobscura. J. Genet., 54, 497-505.

MAYNARD SMITH, J. 1957. Temperature tolerance and acclimatization in Drosophila subobscura. J. Exp. Zool, 34, 85-96. 
MILKMAN, R. 1963. On the mechanism of some temperature effects on Drosophila. J. Gen. Physiol., 46, 1151-1170.

MORRISON, w. w. AND MILKMAN, R. 1978. Modification of heat resistance in Drosophila by selection. Nature, 273, 49-50.

NIKLASSON, M. AND PARKER, E. D., JR. 1994. Fitness variation in an invading parthenogenetic cockroach. Oikos, 71, 47-54.

PARSONS, P. A. 1973. Genetics of resistance to environmental stresses in Drosophila populations. Ann. Rev. Genet., 7, 239-265.

PARSONS, P. A. 1980. Isofemale strains and evolutionary strategies in natural populations. Evol. Biol., 13, $175-217$.
PARSONS, P. A. 1983. The Evolutionary Biology of Colonizing Species. Cambridge University Press, New York.

PARSONS, P. A. 1989. Environmental stresses and conservation of natural populations. Ann. Rev. Ecol. Syst., 20, 29-49.

Quintana, A. AND PREvosti, A. 1990. Genetic and environmental factors in the resistance of Drosophila subobscura adults to high temperature shock 2. Modification of heat resistance by indirect selection. Theor. Appl. Genet., 80, 847-851.

WEIDER, L. J. 1993. A test of the "general-purpose" genotype hypothesis: differential tolerance to thermal and salinity stress among Daphnia clones. Evolution, 47, 965-969. 